\title{
The LightCycler 480 real-time PCR system: a versatile platform for genetic variation research
}

\begin{abstract}
Real-time PCR is a well established technique for studying genetic variation using various probe-based methods for genotyping as well as high-resolution analysis of whole amplicons melted in the presence of saturating DNA dyes. The latter, relatively new, method allows screening for unknown mutations or DNA modifications. The LightCycler 480 real-time PCR system is a multiwell plate-based instrument that provides integrated applications for detecting and characterizing genetic variation using all these methodological approaches.
\end{abstract}

From endpoint fluorescence to melting curve analysis

Today's research on somatic, genetic and epigenetic variation in eukaryotic cells requires fast, accurate and cost-effective methods for screening large numbers of samples or loci in parallel. Variations identified by genomic sequencing or array studies need to be subsequently confirmed and validated.

Real-time PCR has become a well established technology for this purpose. The plate-based LightCycler 480 system offers a broad selection of methods and applications (Fig. 1).

The basic method (endpoint genotyping) is highly suitable for simple experimental setups when well characterized single-nucleotide polymorphisms (SNPs) and regions need to be analyzed, especially when commercial primers and probes are available. Allele-specific signals can be generated and acquired upon the completion of PCR, using two differently labeled hydrolysis probes_ one for each allele — in the same reaction. Primers and probes can be readily combined with LightCycler 480 reagents, and the results can be analyzed with the LightCycler 480 Endpoint Genotyping Software module.

For more advanced setups, melting curves obtained with fluorescent hybridization probes provide additional information about the sequence under study. Known SNPs present in PCR amplicons may be investigated with sequence-specific, labeled probes (known as HybProbe probes) that bind with different strengths to different alleles or allele combinations in the SNP-containing region, depending on whether there are mismatches that keep the probes from fully matching the target sequence. Because signal generation and analysis are done after amplification, there is no influence on PCR efficiency (for example, when DNA amounts or purity are an issue), making the method more robust than endpoint analysis. Only one fluorescence channel is acquired per locus, allowing investi-

\section{Michael Hoffmann, Oliver Geulen \& Christian Weilke}

Roche Applied Science, Nonnenwald 2, 82377 Penzberg, Germany. Correspondence should be addressed to M.H. (michael.hoffmann@roche.com).

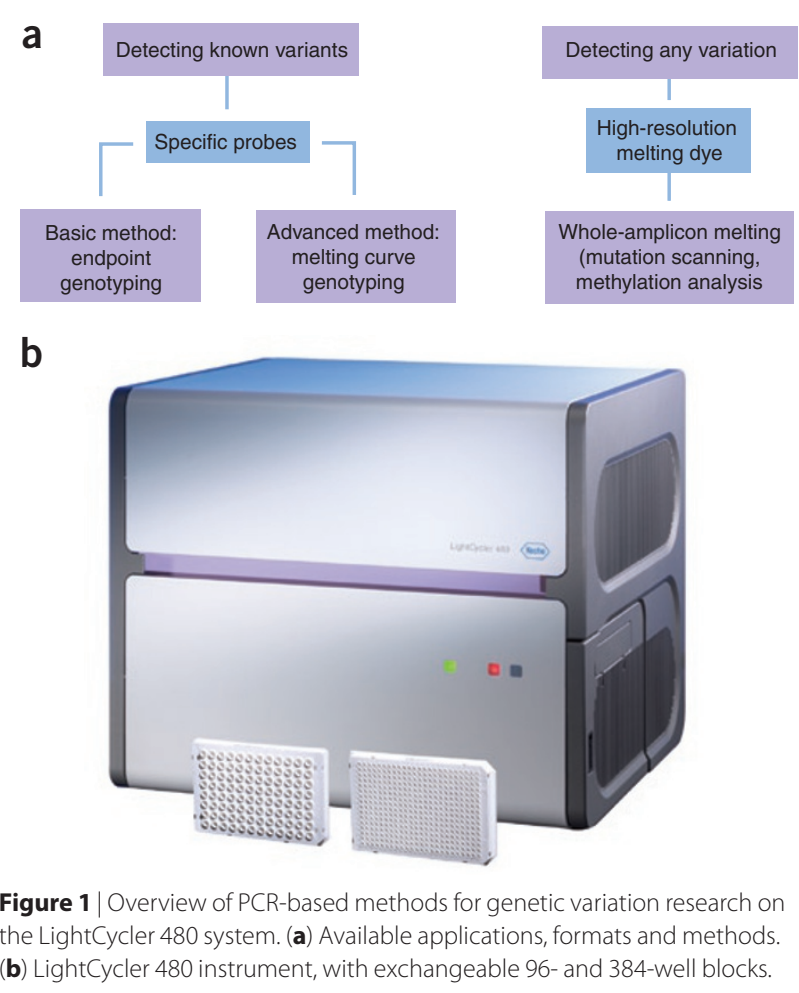

gation of several SNPs in parallel in multiplex assays if a different color is chosen for each SNP. HybProbe probes can also be designed to cover several SNPs under one probe, thus allowing haplotype analysis. Melting curve analysis can also account for unexpected sequence changes present in the investigated region under the probe.

\section{High-resolution melting using ResoLight dye}

When a region being investigated is suspected of containing variants whose exact position is not known, the LightCycler 480 Gene 
a

Normalized and temperature-shifted melting curves

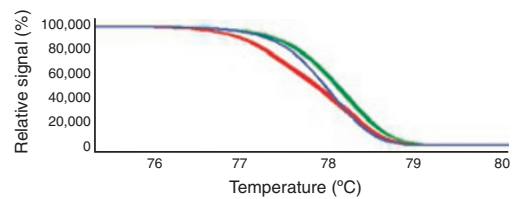

Normalized and temperature-shifted difference plot

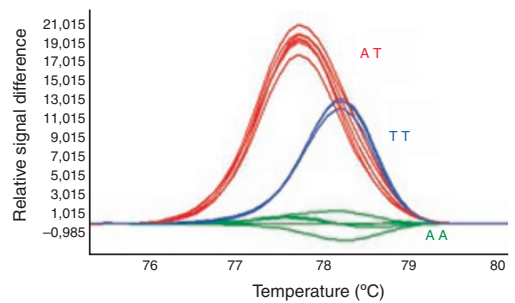

b

Normalized and temperature-shifted melting curves

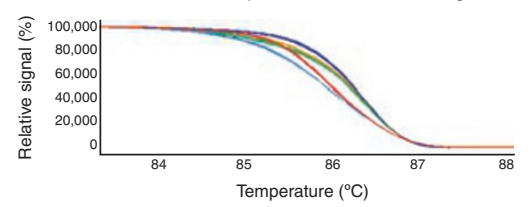

Normalized and temperature-shifted difference plot

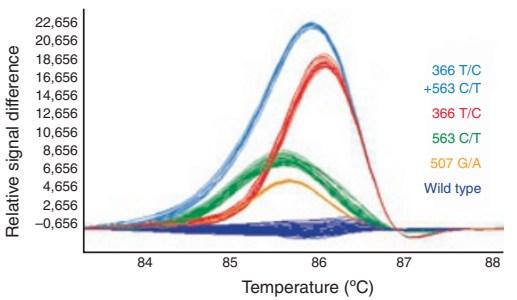

C

Normalized and temperature-shifted melting curves

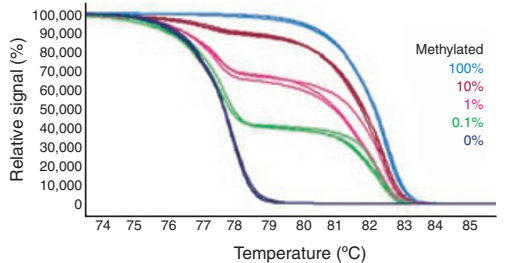

Normalized and temperature-shifted difference plot

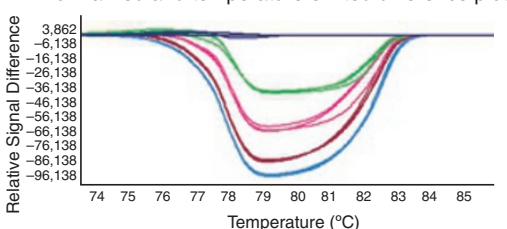

Figure 2 Applications of high-resolution melting analysis on the LightCycler 480 System. (a) A fragment of the TNFSF18 gene with the ATT polymorphism rs723858 was amplified from different samples of human genomic DNA using the LightCycler 480 High Resolution Melting Master and analyzed by high-resolution melting. The LightCycler 480 Gene Scanning Software detects the differences in the melting curves resulting from sequence variations of the PCR products and allocates the samples to groups of the same sequence. The three genotypes are clearly separated, even though A/T polymorphisms, especially the homozygous variants (green and red curves), are hard to distinguish using alternative methods with strand-separation analysis. (b) A section of exon 3 of the CYP2C9 gene was amplified from different samples of human genomic DNA. High-resolution melting analysis revealed groups of samples with different sequence variations. Sequencing of only one sample of each group allowed the sequence determination for all samples. (c) Mixtures with different ratios of genomic DNA from a healthy donor and fully methylated DNA were treated with bisulfite to modify unmethylated cytosines. A fragment of the MGMT tumor suppressor gene was amplified from these treated DNA samples and analyzed by high-resolution melting. Melting curve evaluation allows a rough quantification of the portion of methylated DNA; even samples with only $0.1 \%$ methylated DNA can be clearly distinguished from completely unmethylated DNA. Original data kindly provided by T. K. Wojdacz and A. Dobrovic. (Peter MacCallum Cancer Centre, Melbourne, Australia).

Scanning Software and LightCycler 480 High Resolution Melting Master reagent offer additional benefits. The master mix contains LightCycler 480 ResoLight Dye, a DNA-binding dye whose special binding characteristics allow it to be used at high concentrations without inhibiting PCR. Its homogeneous staining of target sequences results in sharp melting signals, allowing differentiation between homo- and heterozygous samples or often even between homozygous wild-type and mutant samples (Fig. 2a,b). This approach is especially useful as a screening technique before sequencing-for example, as a cost-effective alternative to denaturing high-performance liquid chromatography.

High-resolution melting curve analysis (HRM) on the LightCycler 480 system has greatly simplified discovery and detection of SNPs and enabled a broad range of applications in basic and clinical research-for example, on genes involved in human tumors (Vossen, R. et al. Transferring PCRs to HRM assays on the LightCycler ${ }^{\circledR} 480$ System-examples for BRCA1. Biochemica 4, 10-11; 2007) and malignant hyperthermia ${ }^{1}$ and for pharmacogenetic studies ${ }^{2}$ (Evrard, A. et al. Mutation scanning of the cytidine deaminase gene by highresolution melting curve analysis using the LightCycler ${ }^{\circledR} 480$ system. Biochemica 3, 13-14; 2007). Moreover, HRM has allowed scientists to find new ways to shed light on basic biological mechanisms in which variations at levels other than the DNA primary sequence level are involved. These include RNA editing ${ }^{3}$ and epigenetic changes such as DNA methylation (Fig. 2) (Krypuy, M. et al. Rapid high-throughput methylation analysis using the LightCycler ${ }^{\circledR} 480$ system. Biochemica 1, 11-13; 2008).

\section{Conclusions and outlook}

The LightCycler 480 real-time PCR system is a versatile platform for the study of gene expression regulation and genetic variation ${ }^{4,5}$. Owing to its superior temperature homogeneity, flexible detection channels and powerful software analysis modules, the LightCycler 480 system fulfills the requirements of both basic and advanced genotyping methods.

Further information about the LightCycler 480 System and the mutation detection approaches described is available on the Roche Applied Science special interest page (http://www.lightcycler480.com).

LightCycler and HRM are trademarks of Roche. Patent and license disclaimer information is available online (http://www.lightcycler.com).

1. Grievink, H. \& Stowell, K.M. Identification of ryanodine receptor 1 singlenucleotide polymorphisms by high-resolution melting using the LightCycler 480 System. Anal. Biochem., published online 21 November 2007.

2. Mercier, C. et al. Toxic death case in a patient undergoing gemcitabinebased chemotherapy in relation with cytidine deaminase downregulation. Pharmacogenet. Genomics 17, 841-844 (2007).

3. Chateigner-Boutin, A.L. \& Small, I. A rapid high-throughput method for the detection and quantification of RNA editing based on high-resolution melting of amplicons. Nucleic Acids Res. 35, e114 (2007).

4. Van Eyken, E. et al. A new, easy, and rapid high-throughput detection method for the common GJB2 (CX26), 35delG mutation. Genet. Test. 11, 231-234 (2007)

5. Fazio, G., Palmi, C., Rolink, A., Biondi, A. \& Cazzaniga, G. PAX5/TEL acts as a transcriptional repressor causing down-modulation of CD19, enhances migration to CXCL12, and confers survival advantage in pre-BI cells. Cancer Res. 68, 181-189 (2008).

This article was submitted to Nature Methods by a commercial organization and has not been peer reviewed. Nature Methods takes no responsibility for the accuracy or otherwise of the information provided. 